\title{
On a singular system of fractional nabla difference equations with boundary conditions
}

\author{
Ioannis K Dassios ${ }^{1 *}$ and Dumitru I Baleanu 2,3,4
}

"Correspondence:

jdasios@math.uoa.gr

'school of Mathematics and

Maxwell Institute, The University of

Edinburgh, Mayfield Road,

Edinburgh, EH9 3JZ, United

Kingdom

Full list of author information is

available at the end of the article

\begin{abstract}
In this article, we study a boundary value problem of a class of linear singular systems of fractional nabla difference equations whose coefficients are constant matrices. By taking into consideration the cases that the matrices are square with the leading coefficient matrix singular, square with an identically zero matrix pencil and non-square, we provide necessary and sufficient conditions for the existence and uniqueness of solutions. More analytically, we study the conditions under which the boundary value problem has a unique solution, infinite solutions and no solutions. Furthermore, we provide a formula for the case of the unique solution. Finally, numerical examples are given to justify our theory.
\end{abstract}

Keywords: boundary conditions; singular systems; fractional calculus; nabla operator; difference equations; linear; discrete time system

\section{Introduction}

Difference equations of fractional order have recently proven to be valuable tools in the modeling of many phenomena in various fields of science and engineering. Indeed, we can find numerous applications in viscoelasticity, electrochemistry, control, porous media, electromagnetism, and so forth [1-7]. There has been a significant development in the study of fractional differential/difference equations and inclusions in recent years; see the monographs of Baleanu et al. [1], Kaczorek [4], Klamka et al. [8], Malinowska et al. [5], Podlubny [7], and the survey by Agarwal et al. [9]. For some recent contributions on fractional differential/difference equations, see $[1,4,5,8-27]$ and the references therein. In this article we provide an introductory study for a boundary value problem of a class of singular fractional nabla discrete time systems. If we define $\mathbb{N}_{\alpha}$ by $\mathbb{N}_{\alpha}=\{\alpha, \alpha+1, \alpha+2, \ldots\}$, $\alpha$ integer, and $n$ such that $0<n<1$ or $1<n<2$, then the nabla fractional operator in the case of Riemann-Liouville fractional difference of $n$th order for any $Y_{k}: \mathbb{N}_{a} \rightarrow \mathbb{R}^{m \times 1}$ is defined by, see [5, 10-12, 23],

$$
\nabla_{\alpha}^{n} Y_{k}=\frac{1}{\Gamma(-n)} \sum_{j=\alpha}^{k}(k-j+1)^{\overline{-n-1}} Y_{j},
$$

where the raising power function is defined by

$$
k^{\bar{\alpha}}=\frac{\Gamma(k+\alpha)}{\Gamma(k)} .
$$

C 2013 Dassios and Baleanu; licensee Springer. This is an Open Access article distributed under the terms of the Creative Commons Attribution License (http://creativecommons.org/licenses/by/2.0), which permits unrestricted use, distribution, and reproduction in any medium, provided the original work is properly cited. 
The following problem will then be considered. The singular fractional discrete time systems of the form

$$
F \nabla_{0}^{n} Y_{k}=G Y_{k}, \quad k=1,2, \ldots, N
$$

with known boundary conditions

$$
A_{1} Y_{0}=B_{1}, \quad A_{2} Y_{N}=B_{2}
$$

where $F, G \in \mathcal{M}(r \times m ; \mathcal{F})$ (i.e., the algebra of matrices with elements in the field $\mathcal{F}$ ) with $Y_{k}, V_{k} \in \mathcal{M}(m \times 1 ; \mathcal{F}), A_{1} \in \mathcal{M}\left(r_{1} \times m ; \mathcal{F}\right), A_{2} \in \mathcal{M}\left(r_{2} \times m ; \mathcal{F}\right), B_{1} \in \mathcal{M}\left(r_{1} \times 1 ; \mathcal{F}\right)$ and $B_{2} \in \mathcal{M}\left(r_{2} \times 1 ; \mathcal{F}\right)$. For the sake of simplicity, we set $\mathcal{M}_{m}=\mathcal{M}(m \times m ; \mathcal{F})$ and $\mathcal{M}_{r m}=$ $\mathcal{M}(r \times m ; \mathcal{F})$. The matrices $F$ and $G$ can be non-square (when $r \neq m)$ or square $(r=m)$ and $F$ singular (det $F=0)$. The main purpose will be to provide necessary and sufficient conditions for the existence and uniqueness of solutions of the above boundary value problem, i.e., to study the conditions under which the system has unique, infinite and no solutions and to provide a formula for the case of the unique solution (if it exists). Many authors use matrix pencil theory to study linear discrete time systems with constant matrices; see, for instance, [28-43]. A matrix pencil is a family of matrices $s F-G$, parametrized by a complex number $s$, see $[39,41,44,45]$. When $G$ is square and $F=I_{m}$, where $I_{m}$ is the identity matrix, the zeros of the function $\operatorname{det}(s F-G)$ are the eigenvalues of $G$. Consequently, the problem of finding the nontrivial solutions of the equation

$$
s F X=G X
$$

is called the generalized eigenvalue problem. Although the generalized eigenvalue problem looks like a simple generalization of the usual eigenvalue problem, it exhibits some important differences. In the first place, it is possible for $F, G$ to be non-square matrices. Moreover, even with $F, G$ square it is possible (in the case $F$ is singular) for $\operatorname{det}(s F-G$ ) to be identically zero, independent of $s$. Finally, even if we assume $F, G$ square matrices with a non-zero pencil, it is possible (when $F$ is singular) for the problem to have infinite eigenvalues. To see this, write the generalized eigenvalue problem in the reciprocal form

$$
F X=s^{-1} G X \text {. }
$$

If $F$ is singular with a null vector $X$, then $F X=0_{m, 1}$, so that $X$ is an eigenvector of the reciprocal problem corresponding to eigenvalue $s^{-1}=0$; i.e., $s=\infty$.

Definition 1.1 Given $F, G \in \mathcal{M}_{r m}$ and an arbitrary $s \in F$, the matrix pencil $s F-G$ is called:

1. Regular when $r=m$ and $\operatorname{det}(s F-G) \neq 0$.

2. Singular when $r \neq m$ or $r=m$ and $\operatorname{det}(s F-G) \equiv 0$.

The paper is organized as follows. In Section 2, we study the existence of solutions of the system (1) when its pencil is regular. In Section 3 we study the case of the system (1) with a singular pencil, and Section 3 contains numerical examples. 


\section{Regular case}

In this section, we consider the case of the system (1) with a regular pencil. The class of $s F-G$ is characterized by a uniquely defined element, known as complex Weierstrass canonical form, $s F_{w}-Q_{w}$, see $[39,41,44,45]$, specified by the complete set of invariants of $s F-G$. This is the set of elementary divisors (e.d.) obtained by factorizing the invariant polynomials into powers of homogeneous polynomials irreducible over the field $\mathcal{F}$. In the case where $s F-G$ is regular, we have e.d. of the following type:

- e.d. of the type $\left(s-a_{j}\right)^{p_{j}}$ are called finite elementary divisors (f.e.d.), where $a_{j}$ is a finite eigenvalue of algebraic multiplicity $p_{j}$;

- e.d. of the type $\hat{s}^{q}=\frac{1}{s^{q}}$ are called infinite elementary divisors (i.e.d.), where $q$ is the algebraic multiplicity of the infinite eigenvalues.

We assume that $\sum_{i=1}^{v} p_{j}=p$ and $p+q=m$.

Definition 2.1 Let $B_{1}, B_{2}, \ldots, B_{l}$ be elements of $\mathcal{M}_{m}$. The direct sum of them denoted by $B_{1} \oplus B_{2} \oplus \cdots \oplus B_{l}$ is the blockdiag $\left[B_{1} B_{2} \cdots B_{l}\right]$.

From the regularity of $s F-G$, there exist nonsingular matrices $P, Q \in \mathcal{M}_{m}$ such that

$$
P F Q=F_{w}=I_{p} \oplus H_{q}
$$

and

$$
P G Q=G_{w}=J_{p} \oplus I_{q} .
$$

The complex Weierstrass form $s F_{w}-Q_{w}$ of the regular pencil $s F-G$ is defined by

$$
s F_{w}-Q_{w}:=s I_{p}-J_{p} \oplus s H_{q}-I_{q},
$$

where the first normal Jordan-type element is uniquely defined by the set of the finite eigenvalues of $s F-G$ and has the form

$$
s I_{p}-J_{p}:=s I_{p_{1}}-J_{p_{1}}\left(a_{1}\right) \oplus \cdots \oplus s I_{p_{v}}-J_{p_{v}}\left(a_{v}\right) .
$$

The second uniquely defined block $s H_{q}-I_{q}$ corresponds to the infinite eigenvalues of $s F-$ $G$ and has the form

$$
s H_{q}-I_{q}:=s H_{q_{1}}-I_{q_{1}} \oplus \cdots \oplus s H_{q_{\sigma}}-I_{q_{\sigma}} .
$$

The matrix $H_{q}$ is a nilpotent element of $\mathcal{M}_{q}$ with index $q^{*}=\max \left\{q_{j}: j=1,2, \ldots, \sigma\right\}$, where

$$
H_{q}^{q^{*}}=0_{q, q}
$$

and $I_{p_{j}}, J_{p_{j}}\left(a_{j}\right), H_{q_{j}}$ are defined as

$$
I_{p_{j}}=\left[\begin{array}{ccccc}
1 & 0 & \cdots & 0 & 0 \\
0 & 1 & \cdots & 0 & 0 \\
\vdots & \vdots & \ddots & \vdots & \vdots \\
0 & 0 & \cdots & 0 & 1
\end{array}\right] \in \mathcal{M}_{p_{j}},
$$




$$
\begin{aligned}
& J_{p_{j}}\left(a_{j}\right)=\left[\begin{array}{ccccc}
a_{j} & 1 & \cdots & 0 & 0 \\
0 & a_{j} & \cdots & 0 & 0 \\
\vdots & \vdots & \ddots & \vdots & \vdots \\
0 & 0 & \cdots & a_{j} & 1 \\
0 & 0 & \cdots & 0 & a_{j}
\end{array}\right] \in \mathcal{M}_{p_{j}}, \\
& H_{q_{j}}=\left[\begin{array}{ccccc}
0 & 1 & \cdots & 0 & 0 \\
0 & 0 & \cdots & 0 & 0 \\
\vdots & \vdots & \ddots & \vdots & \vdots \\
0 & 0 & \cdots & 0 & 1 \\
0 & 0 & \cdots & 0 & 0
\end{array}\right] \in \mathcal{M}_{q_{j}} .
\end{aligned}
$$

For algorithms about the computations of the Jordan matrices, see [39, 41, 44, 45].

Definition 2.2 If for the system (1) with boundary conditions (2) there exists at least one solution, the boundary value problem (1)-(2) is said to be consistent.

For the regular matrix pencil of the system (1), there exist nonsingular matrices $P, Q \in$ $\mathcal{M}_{m}$ as applied in (3), (4). Let

$$
Q=\left[\begin{array}{ll}
Q_{p} & Q_{q}
\end{array}\right]
$$

where $Q_{p} \in \mathcal{M}_{m p}$ is a matrix with columns $p$ linear independent (generalized) eigenvectors of the $p$ finite eigenvalues of $s F-G$, and $Q_{q} \in \mathcal{M}_{m q}$ is a matrix with columns $q$ linear independent (generalized) eigenvectors of the $q$ infinite eigenvalues of $s F-G$.

Lemma 2.1 Consider the system (1) with a regular pencil. Then the system (1) is divided into two subsystems:

$$
\nabla_{0}^{n} Z_{k}^{p}=J_{p} Z_{k}^{p}
$$

and

$$
H_{q} \nabla_{0}^{n} Z_{k}^{q}=Z_{k}^{q}
$$

Proof Consider the transformation

$$
Y_{k}=Q Z_{k}
$$

and by substituting (6) into (1), we obtain

$$
F \nabla_{0}^{n} Q Z_{k}=G Q Z_{k}
$$

or, equivalently,

$$
F Q \nabla_{0}^{n} Z_{k}=G Q Z_{k}
$$


Whereby multiplying by $P$, we arrive at

$$
F_{w} \nabla_{0}^{n} Z_{k}=G_{w} Z_{k}
$$

Moreover, let

$$
Z_{k}=\left[\begin{array}{c}
Z_{k}^{p} \\
Z_{k}^{q}
\end{array}\right]
$$

where $Z_{k}^{p} \in \mathcal{M}_{p 1}, Z_{k}^{q} \in \mathcal{M}_{q 1}$, and by using (3) and (4), we obtain

$$
\left[\begin{array}{cc}
I_{p} & 0_{p, q} \\
0_{q, p} & H_{q}
\end{array}\right] \nabla_{\alpha}^{n}\left[\begin{array}{c}
Z_{k}^{p} \\
Z_{k}^{q}
\end{array}\right]=\left[\begin{array}{cc}
J_{p} & 0_{p, q} \\
0_{q, p} & I_{q}
\end{array}\right]\left[\begin{array}{c}
Z_{k}^{p} \\
Z_{k}^{q}
\end{array}\right] .
$$

From the above expressions, we arrive easily at the subsystems

$$
\nabla_{0}^{n} Z_{k}^{p}=J_{p} Z_{k}^{p}
$$

and

$$
H_{q} \nabla_{0}^{n} Z_{k}^{q}=Z_{k}^{q}
$$

The proof is completed.

Definition 2.3 With $F_{n, n}\left(J_{p}(k+n)^{\bar{n}}\right)$ we denote the discrete Mittag-Leffler function with two parameters defined by

$$
F_{n, n}\left(J_{p}(k+n)^{\bar{n}}\right)=\sum_{i=0}^{\infty} J_{p}^{i} \frac{(k+n)^{\overline{i n}}}{\Gamma((i+1) n)} .
$$

See $[10-12,23,46]$.

Proposition 2.1 The subsystem (7) has the solution

$$
Z_{k}^{p}=(k+1)^{\overline{n-1}} F_{n, n}\left(J_{p}(k+n)^{\bar{n}}\right)\left(I_{p}-J_{p}\right) Z_{0}^{p}
$$

if and only if

$$
\left\|J_{p}\right\|<1
$$

where $\|\cdot\|$ is an induced matrix norm and $F_{n, n}\left(J_{p}(k+n)^{\bar{n}}\right)$ is the discrete Mittag-Leffler function with two parameters as defined by Definition 2.3.

Proof From $[10-12,23,46]$ the solution of (7) can be calculated and given by the formula

$$
Z_{k}^{p}=(k+1)^{\overline{n-1}} F_{n, n}\left(J_{p}(k+n)^{\bar{n}}\right)\left(I_{p}-J_{p}\right) Z_{0}^{p}
$$


or, equivalently, by

$$
Z_{k}^{p}=(k+1)^{\overline{n-1}}\left(\sum_{i=0}^{\infty} J_{p}^{i} \frac{(k+n)^{\overline{i n}}}{\Gamma((i+1) n)}\right)\left(I_{p}-J_{p}\right) Z_{0}^{p} .
$$

The existence and uniqueness of the above solution depends on the convergence of the matrix power series

$$
\sum_{i=0}^{\infty} J_{p}^{i} \frac{(k+n)^{\overline{i n}}}{\Gamma((i+1) n)}
$$

or, equivalently, if and only if

$$
\lim _{i \rightarrow \infty} \frac{\left\|J_{p}^{(i+1)} \frac{(k+n)^{\overline{(i+1) n}}}{\Gamma((i+2) n)}\right\|}{\left\|J_{p}^{i} \frac{(k+n)^{\bar{n}}}{\Gamma((i+1) n)}\right\|}<1
$$

or, equivalently,

$$
\left\|J_{p}\right\|<\lim _{i \rightarrow \infty} \frac{\frac{(k+n)^{\overline{\bar{n}}}}{\Gamma((i+1) n)}}{\frac{(k+n) \overline{(i+1) n}}{\Gamma((i+2) n)}} .
$$

By using the property

$$
\Gamma(z+1)=z \Gamma(z)
$$

we get

$$
\left\|J_{p}\right\|<\lim _{i \rightarrow \infty} \frac{((k-1)+(i+1) n) \cdots(1+(i+1) n)((i+1) n)}{((k-1)+(i+2) n) \cdots(1+(i+2) n)((i+2) n)}
$$

or, equivalently,

$$
\left\|J_{p}\right\|<1
$$

The proof is completed.

Proposition 2.2 The subsystem (8) has the unique solution

$$
Z_{k}^{q}=0_{q, 1} .
$$

Proof Let $q_{*}$ be the index of the nilpotent matrix $H_{q}$, i.e., $H_{q}^{q_{*}}=0_{q, q}$. Then if we obtain the following equations:

$$
\begin{aligned}
& H_{q} \nabla_{0}^{n} Z_{k}^{q}=Z_{k}^{q}, \\
& H_{q}^{2} \nabla_{0}^{2 n} Z_{k}^{q}=H_{q} \nabla_{0}^{n} Z_{k}^{q}, \\
& H_{q}^{3} \nabla_{0}^{3 n} Z_{k}^{q}=H_{q}^{2} \nabla_{0}^{2 n} Z_{k}^{q},
\end{aligned}
$$




$$
\begin{aligned}
& H_{q}^{4} \nabla_{0}^{4 n} Z_{k}^{q}=H_{q}^{3} \nabla_{0}^{3 n} Z_{k}^{q}, \\
& \vdots \\
& H_{q}^{q_{*}-1} \nabla_{0}^{\left(q_{*}-1\right) n} Z_{k}^{q}=H_{q}^{q_{*}-2} \nabla_{0}^{\left(q_{*}-2\right) n} Z_{k}^{q}, \\
& H_{q}^{q_{*}} \nabla_{0}^{q_{*} n} Z_{k}^{q}=H_{q}^{q_{*}-1} \nabla_{0}^{\left(q_{*}-1\right) n} Z_{k}^{q}
\end{aligned}
$$

by taking the sum of the above equations and using the fact that $H_{q}^{q^{*}}=0_{q, q}$, we arrive easily at the solution (12). The proof is completed.

Theorem 2.1 Consider the system (1) with a regular pencil and boundary conditions of type (2). Then the boundary value problem (1)-(2) is consistent if and only if:

1. The pencil $s F-G$ has $p$ distinct eigenvalues and all lie within the open disk

$|s|<1$

2.

$$
\left[\begin{array}{l}
B_{1} \\
B_{2}
\end{array}\right] \in \text { colspan }\left[\begin{array}{c}
A_{1} Q_{p} \\
A_{2} Q_{p}(N+1)^{\overline{n-1}} F_{n, n}\left(J_{p}(N+n)^{\bar{n}}\right)\left(I_{p}-J_{p}\right)
\end{array}\right] .
$$

Furthermore, when the boundary value problem (1)-(2) is consistent, it has a unique solution if and only if:

1.

$$
p \leq r_{1}+r_{2}
$$

2.

$$
\operatorname{rank}\left[\begin{array}{c}
A_{1} Q_{p} \\
A_{2} Q_{p}(N+1)^{\overline{n-1}} F_{n, n}\left(I_{p}(N+n)^{\bar{n}}\right)\left(I_{p}-J_{p}\right)
\end{array}\right]=p .
$$

In this case the unique solution is then given by

$$
Y_{k}=Q_{p}(k+1)^{\overline{n-1}} F_{n, n}\left(J_{p}(k+n)^{\bar{n}}\right)\left(I_{p}-J_{p}\right) C,
$$

where $C$ is the unique solution of the algebraic system

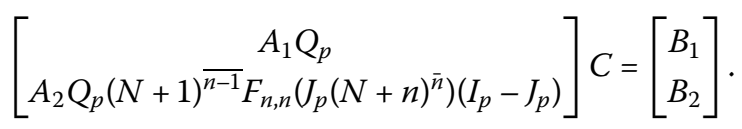

Proof By applying the transformation (6) into the system (1), we get the systems (7), (8) with solutions (10), (12) respectively. Note that from Proposition 2.1 the solution (10) exists if and only if

$$
\left\|J_{p}\right\|<1
$$


where $J_{p}$ is the Jordan matrix related to the $p$ finite eigenvalues of the pencil $s F-G$, which is equivalent to the fact that the finite eigenvalues of the pencil must be distinct and all lie within the unit disk $|s|<1$. Based on these results, the solution of (1) can be written as

$$
Y_{k}=Q Z_{k}=\left[\begin{array}{ll}
Q_{p} & Q_{q}
\end{array}\right]\left[\begin{array}{c}
Z_{k}^{p} \\
Z_{k}^{q}
\end{array}\right]
$$

or, equivalently,

$$
Y_{k}=Q_{p} Z_{k}^{p}+Q_{q} Z_{k}^{q}
$$

or, equivalently, by using (10), (12)

$$
Y_{k}=Q_{p}(k+1)^{\overline{n-1}} F_{n, n}\left(J_{p}(k+n)^{\bar{n}}\right)\left(I_{p}-J_{p}\right) Z_{0}^{p} .
$$

The initial value $Z_{0}^{p}$ of the subsystem (7) is not known and can be replaced by a constant vector $C \in \mathcal{M}_{p 1}$

$$
Y_{k}=Q_{p}(k+1)^{\overline{n-1}} F_{n, n}\left(J_{p}(k+n)^{\bar{n}}\right)\left(I_{p}-J_{p}\right) C .
$$

The above solution exists if and only if

$$
A_{1} Y_{0}=B_{1}, \quad A_{2} Y_{N}=B_{2}
$$

or, equivalently,

$$
A_{1} Q_{p} C=B_{1}, \quad A_{2} Q_{p}(N+1)^{\overline{n-1}} F_{n, n}\left(J_{p}(N+n)^{\bar{n}}\right)\left(I_{p}-J_{p}\right) C=B_{2},
$$

or, equivalently,

$$
\left[\begin{array}{c}
A_{1} Q_{p} \\
A_{2} Q_{p}(N+1)^{\frac{\overline{n-1}}{F_{n, n}}}\left(J_{p}(N+n)^{\bar{n}}\right)\left(I_{p}-J_{p}\right)
\end{array}\right] C=\left[\begin{array}{l}
B_{1} \\
B_{2}
\end{array}\right] .
$$

For the above algebraic system, there exists at least one solution if and only if

$$
\left[\begin{array}{l}
B_{1} \\
B_{2}
\end{array}\right] \in \operatorname{colspan}\left[\begin{array}{c}
A_{1} Q_{p} \\
A_{2} Q_{p}(N+1)^{\overline{n-1}} F_{n, n}\left(I_{p}(N+n)^{\bar{n}}\right)\left(I_{p}-J_{p}\right)
\end{array}\right] .
$$

The algebraic system (17) contains $r_{1}+r_{2}$ equations and $p$ unknowns. Hence the solution is unique if and only if

$$
p \leq r_{1}+r_{2}
$$

and

$$
\operatorname{rank}\left[\begin{array}{c}
A_{1} Q_{p} \\
A_{2} Q_{p}(N+1)^{\overline{n-1}} F_{n, n}\left(J_{p}(N+n)^{\bar{n}}\right)\left(I_{p}-J_{p}\right)
\end{array}\right]=p,
$$


where $C$ is then the unique solution of (17). This can be proved as follows. If we assume that the algebraic system has two solutions $C_{1}$ and $C_{2}$, then

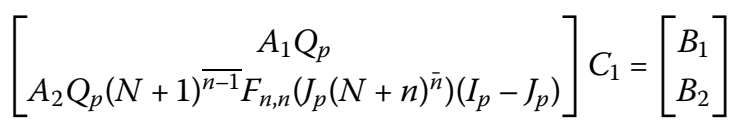

and

$$
\left[\begin{array}{c}
A_{1} Q_{p} \\
A_{2} Q_{p}(N+1)^{\overline{n-1}} F_{n, n}\left(J_{p}(N+n)^{\bar{n}}\right)\left(I_{p}-J_{p}\right)
\end{array}\right] C_{2}=\left[\begin{array}{c}
B_{1} \\
B_{2}
\end{array}\right]
$$

or, equivalently,

$$
\left[\begin{array}{c}
A_{1} Q_{p} \\
A_{2} Q_{p}(N+1)^{\overline{n-1}} F_{n, n}\left(J_{p}(N+n)^{\bar{n}}\right)\left(I_{p}-J_{p}\right)
\end{array}\right]\left(C_{1}-C_{2}\right)=0_{p, 1} .
$$

But the matrix $\left[\begin{array}{c}A_{1} Q_{p} \\ A_{2} Q_{p}(N+1)^{\frac{1}{n-1}} F_{n, n}\left(J_{p}(N+n)^{\bar{n}}\right)\left(I_{p}-J_{p}\right)\end{array}\right]$ is left invertible since it is assumed to have $p$ linear independent columns and $r_{1}+r_{2} \geq p$ and hence

$$
C_{1}=C_{2} .
$$

The unique solution is then given from (16). The proof is completed.

\section{Singular case}

In this section, we consider the case of the system (1) with a singular pencil. The class of $s F-G$ in this case is characterized by a uniquely defined element, $s F_{K}-Q_{K}$, known as the complex Kronecker canonical form, see [39, 41, 44, 45], specified by the complete set of invariants of the singular pencil $s F-G$. This is the set of the elementary divisors (e.d.) and the minimal indices (m.i.). Unlike the case of the regular pencils, where the pencil is characterized only from the e.d., the characterization of a singular matrix pencil apart from the set of the determinantal divisors requires the definition of additional sets of invariants, the minimal indices. The distinguishing feature of a singular pencil $s F-G$ is that either $r \neq m$ or $r=m$ and $\operatorname{det}(s F-G) \equiv 0$. Let $\mathcal{N}_{r}, \mathcal{N}_{l}$ be the right and the left null space of a matrix respectively. Then the equations

$$
\begin{aligned}
& (s F-G) U(s)=0_{r, 1}, \\
& V^{T}(s)(s F-G)=0_{1, m},
\end{aligned}
$$

where ()$^{T}$ is the transpose tensor, have solutions in $U(s), V(s)$, which are vectors in the rational vector spaces $\mathcal{N}_{r}(s F-G)$ and $\mathcal{N}_{l}(s F-G)$ respectively. The binary vectors $U(s)$ and $V^{T}(s)$ express dependence relationships among the columns or rows of $s F-G$ respectively. Note that $U(s) \in \mathcal{M}_{m 1}$ and $V(s) \in \mathcal{M}_{r 1}$ are polynomial vectors. Let $d=\operatorname{dim} \mathcal{N}_{r}(s F-G)$ and $t=\operatorname{dim} \mathcal{N}_{l}(s F-G)$. It is known, see $[39,41,44,45]$, that $\mathcal{N}_{r}(s F-G)$ and $\mathcal{N}_{l}(s F-G)$ as rational vector spaces are spanned by minimal polynomial bases of minimal degrees

$$
\epsilon_{1}=\epsilon_{2}=\cdots=\epsilon_{g}=0<\epsilon_{g+1} \leq \cdots \leq \epsilon_{d}
$$


and

$$
\zeta_{1}=\zeta_{2}=\cdots=\zeta_{h}=0<\zeta_{h+1} \leq \cdots \leq \zeta_{t}
$$

respectively. The set of minimal indices $\epsilon_{1}, \epsilon_{2}, \ldots, \epsilon_{d}$ and $\zeta_{1}, \zeta_{2}, \ldots, \zeta_{t}$ are known as column minimal indices (c.m.i.) and row minimal indices (r.m.i.) of $s F-G$ respectively. To sum up, in the case of a singular pencil, we have invariants of the following type:

- finite elementary divisors of the type $\left(s-a_{j}\right)^{p_{j}}$;

- infinite elementary divisors of the type $\hat{s}^{q}=\frac{1}{s^{q}}$;

- column minimal indices of the type $\epsilon_{1}=\epsilon_{2}=\cdots=\epsilon_{g}=0<\epsilon_{g+1} \leq \cdots \leq \epsilon_{d}$;

- row minimal indices of the type $\zeta_{1}=\zeta_{2}=\cdots=\zeta_{h}=0<\zeta_{h+1} \leq \cdots \leq \zeta_{t}$.

The Kronecker canonical form, see [39, 41, 44, 45], is defined by

$$
s F_{K}-G_{K}:=s I_{p}-J_{p} \oplus s H_{q}-I_{q} \oplus s F_{\epsilon}-G_{\epsilon} \oplus s F_{\zeta}-G_{\zeta} \oplus 0_{h, g}
$$

where $s I_{p}-J_{p}, s H_{q}-I_{q}$ are defined as in Section 2. The matrices $F_{\epsilon}, G_{\epsilon}, F_{\zeta}$ and $G_{\zeta}$ are defined by

$$
F_{\epsilon}=\operatorname{blockdiag}\left\{L_{\epsilon_{g+1}}, L_{\epsilon_{g+2}}, \ldots, L_{\epsilon_{d}}\right\}
$$

where $\left.L_{\epsilon}={ }_{I_{\epsilon}} \vdots 0_{\epsilon, 1}\right]$ for $\epsilon=\epsilon_{g+1}, \ldots, \epsilon_{d}$

$$
G_{\epsilon}=\operatorname{blockdiag}\left\{\bar{L}_{\epsilon_{g+1}}, \bar{L}_{\epsilon_{g+2}}, \ldots, \bar{L}_{\epsilon_{d}}\right\}
$$

where $\bar{L}_{\epsilon}=\left[{ }_{0_{\epsilon, 1}} \vdots_{I_{\epsilon}}\right]$ for $\epsilon=\epsilon_{g+1}, \ldots, \epsilon_{d}$. The matrices $F_{\zeta}, G_{\zeta}$ are defined as

$$
F_{\zeta}=\operatorname{blockdiag}\left\{L_{\zeta_{h+1}}, L_{\zeta_{h+2}}, \ldots, L_{\zeta t}\right\}
$$

where $L_{\zeta}=\left[\begin{array}{c}I_{\zeta} \\ 0_{1, \zeta}\end{array}\right]$ for $\zeta=\zeta_{h+1}, \ldots, \zeta_{t}$

$$
G_{\zeta}=\operatorname{blockdiag}\left\{\bar{L}_{\zeta_{h+1}}, \bar{L}_{\zeta_{h+2}}, \ldots, \bar{L}_{\zeta t}\right\}
$$

where $\bar{L}_{\zeta}=\left[\begin{array}{c}0_{1, \zeta} \\ I_{\zeta}\end{array}\right]$ for $\zeta=\zeta_{h+1}, \ldots, \zeta_{t}$.

For algorithms about the computations of these matrices, see [39, 41, 44, 45].

Following the above given analysis, there exist nonsingular matrices $P, Q$ with $P \in \mathcal{M}_{r}$, $Q \in \mathcal{M}_{m}$ such that

$$
\begin{aligned}
& P F Q=F_{K}, \\
& P G Q=G_{K} .
\end{aligned}
$$

Let

$$
Q=\left[\begin{array}{lllll}
Q_{p} & Q_{q} & Q_{\epsilon} & Q_{\zeta} & Q_{g}
\end{array}\right]
$$

where $Q_{p} \in \mathcal{M}_{m p}, Q_{q} \in \mathcal{M}_{m q}, Q_{\epsilon} \in \mathcal{M}_{m(d-g)}, Q_{\zeta} \in \mathcal{M}_{m(t-h)}$ and $Q_{g} \in \mathcal{M}_{m g}$. 
Lemma 3.1 The system (1) is divided into five subsystems:

$$
\nabla_{0}^{n} Z_{k}^{p}=J_{p} Z_{k}^{p}
$$

the subsystem

$$
H_{q} \nabla_{0}^{n} Z_{k}^{q}=Z_{k}^{q}
$$

the subsystem

$$
F_{\epsilon} \nabla_{0}^{n} Z_{k}^{\epsilon}=G_{\epsilon} Z_{k}^{\epsilon}
$$

the subsystem

$$
F_{\zeta} \nabla_{0}^{n} Z_{k}^{\zeta}=G_{\zeta} Z_{k}^{\zeta}
$$

and the subsystem

$$
0_{h, g} \cdot \nabla_{0}^{n} Z_{k+1}^{g}=0_{h, g} \cdot Z_{k}^{g} .
$$

Proof Consider the transformation

$$
Y_{k}=Q Z_{k}
$$

Substituting the previous expression into (1), we obtain

$$
F Q \nabla_{0}^{n} Z_{k}=G Q Z_{k}
$$

Whereby multiplying by $P$ and using (27), we arrive at

$$
F_{K} \nabla_{0}^{n} Z_{k}=G_{K} Z_{k}
$$

Moreover, let

$$
Z_{k}=\left[\begin{array}{c}
Z_{k}^{p} \\
Z_{k}^{q} \\
Z_{k}^{\epsilon} \\
Z_{k}^{\zeta} \\
Z_{k}^{g}
\end{array}\right],
$$

where $Z_{p}^{k} \in \mathcal{M}_{p 1}, Z_{q}^{k} \in \mathcal{M}_{q 1}, Z_{\epsilon}^{k} \in \mathcal{M}_{(d-g) 1}, Z_{\zeta}^{k} \in \mathcal{M}_{(t-h) 1}$ and $Z_{g}^{k} \in \mathcal{M}_{g 1}$. Taking into account the above expressions, we arrive easily at the subsystems (29), (30), (31), (32), and (33). The proof is completed.

Solving the system (1) is equivalent to solving subsystems (29), (30), (31), (32) and (33). The solutions of the systems (29), (30) are given by (10) and (12) respectively; see Propositions 2.1 and 2.2 . 
Proposition 3.1 The subsystem (31) has infinite solutions and can be taken arbitrarily

$$
Z_{k}^{\epsilon}=C_{k, 1}
$$

Proof If we set

$$
Z_{k}^{\epsilon}=\left[\begin{array}{c}
Z_{k}^{\epsilon_{g+1}} \\
Z_{k}^{\epsilon_{g+2}} \\
\vdots \\
Z_{k}^{\epsilon_{d}}
\end{array}\right]
$$

by using (23), (24), the system (31) can be written as

$$
\operatorname{blockdiag}\left\{L_{\epsilon_{g+1}}, \ldots, L_{\epsilon_{d}}\right\}\left[\begin{array}{c}
\nabla_{0}^{n} Z_{k}^{\epsilon_{g+1}} \\
\nabla_{0}^{n} Z_{k}^{\epsilon_{+2}} \\
\vdots \\
\nabla_{0}^{n} Z_{k}^{\epsilon_{d}}
\end{array}\right]=\operatorname{blockdiag}\left\{\bar{L}_{\epsilon_{g+1}}, \ldots, \bar{L}_{\epsilon_{d}}\right\}\left[\begin{array}{c}
Z_{k}^{\epsilon_{g+1}} \\
Z_{k}^{\epsilon_{g+2}} \\
\vdots \\
Z_{k}^{\epsilon_{d}}
\end{array}\right] .
$$

Then, for the non-zero blocks, a typical equation from (37) can be written as

$$
L_{\epsilon_{i}} \nabla_{0}^{n} Z_{k}^{\epsilon_{i}}=\bar{L}_{\epsilon_{i}} Z_{k}^{\epsilon_{i}}, \quad i=g+1, g+2, \ldots, d
$$

or, equivalently,

$$
\left[\begin{array}{lll}
I_{\epsilon_{i}} & \vdots & 0_{\epsilon_{i}, 1}
\end{array}\right] \nabla_{0}^{n} Z_{k}^{\epsilon_{i}}=\left[0_{\epsilon_{i}, 1} \quad \vdots \quad I_{\epsilon_{i}}\right] Z_{k}^{\epsilon_{i}},
$$

or, equivalently,

$$
\left[\begin{array}{ccccc}
1 & 0 & \cdots & 0 & 0 \\
0 & 1 & \cdots & 0 & 0 \\
\vdots & \vdots & \cdots & \vdots & \vdots \\
0 & 0 & \cdots & 1 & 0
\end{array}\right]\left[\begin{array}{c}
\nabla_{0}^{n} z_{k}^{\epsilon_{i}, 1} \\
\nabla_{0}^{n} z_{k}^{\epsilon_{i, 2}} \\
\vdots \\
\nabla_{0}^{n} z_{k}^{\epsilon_{i}, \epsilon_{i}} \\
\nabla_{0}^{n} z_{k}^{\epsilon_{i}, \epsilon_{i}+1}
\end{array}\right]=\left[\begin{array}{ccccc}
0 & 1 & \cdots & 0 & 0 \\
0 & 0 & \cdots & 0 & 0 \\
\vdots & \vdots & \cdots & \vdots & \vdots \\
0 & 0 & \cdots & 0 & 1
\end{array}\right]\left[\begin{array}{c}
z_{k}^{\epsilon_{i}, 1} \\
z_{k}^{\epsilon_{i, 2}} \\
\vdots \\
z_{k}^{\epsilon_{i}, \epsilon_{i}} \\
z_{k}^{\epsilon_{i}, \epsilon_{i}+1}
\end{array}\right],
$$

or, equivalently,

$$
\begin{aligned}
& \nabla_{0}^{n} z_{k}^{\epsilon_{i}, 1}=z_{k}^{\epsilon_{i}, 2}+v_{k}^{\epsilon_{i}, 1}, \\
& \nabla_{0}^{n} z_{k}^{\epsilon_{i}, 2}=z_{k}^{\epsilon_{i}, 3}+v_{k}^{\epsilon_{i}, 2}, \\
& \vdots \\
& \nabla_{0}^{n} z_{k}^{\epsilon_{i}, \epsilon_{i}}=\nabla_{0}^{n} z_{k}^{\epsilon_{i}, \epsilon_{i}+1} .
\end{aligned}
$$

The system (39) is a regular-type system of difference equations with $\epsilon_{i}$ equations and $\epsilon_{i}+1$ unknowns. It is clear from the above analysis that in every one of the $d-g$ subsystems one 
of the coordinates of the solution has to be arbitrary by assigned total. The solution of the system can be assigned arbitrarily

$$
Z_{k}^{\epsilon}=C_{k, 1}
$$

The proof is completed.

Proposition 3.2 The solution of the system (32) is unique and is the zero solution

$$
Z_{k}^{\zeta}=0_{t-h, 1}
$$

Proof From (25), (26) the subsystem (32) can be written as

$$
\operatorname{blockdiag}\left\{L_{\zeta_{h+1}}, \ldots, L_{\zeta t}\right\}\left[\begin{array}{c}
\nabla_{0}^{n} Z_{k}^{\zeta+1} \\
\nabla_{0}^{n} Z_{k}^{\zeta h+2} \\
\vdots \\
\nabla_{0}^{n} Z_{k}^{\zeta t}
\end{array}\right]=\operatorname{blockdiag}\left\{\bar{L}_{\zeta h+1}, \ldots, \bar{L}_{\zeta t}\right\}\left[\begin{array}{c}
Z_{k}^{\zeta h+1} \\
Z_{k}^{\zeta h+2} \\
\vdots \\
Z_{k}^{\zeta t}
\end{array}\right]
$$

Then for the non-zero blocks, a typical equation from (41) can be written as

$$
L_{\zeta j} \nabla_{0}^{n} Z_{k}^{\zeta_{j}}=\bar{L}_{\zeta j} Z_{k}^{\zeta_{j}}, \quad j=h+1, h+2, \ldots, t
$$

or, equivalently,

$$
\left[\begin{array}{c}
I_{\zeta_{j}} \\
\cdots \\
0_{1, \zeta_{j}}
\end{array}\right] \nabla_{0}^{n} Z_{k}^{\zeta_{j}}=\left[\begin{array}{c}
0_{1, \zeta_{j}} \\
\cdots \\
I_{\zeta_{j}}
\end{array}\right] Z_{k}^{\zeta_{j}},
$$

or, equivalently,

$$
\left[\begin{array}{cccc}
1 & 0 & \cdots & 0 \\
0 & 1 & \cdots & 0 \\
\vdots & \vdots & \cdots & \vdots \\
0 & 0 & \cdots & 1 \\
0 & 0 & \cdots & 0
\end{array}\right]\left[\begin{array}{c}
\nabla_{0}^{n} z_{k}^{\zeta_{j}, 1} \\
\nabla_{0}^{n} z_{k}^{\zeta_{j}, 2} \\
\vdots \\
\nabla_{0}^{n} z_{k}^{\zeta_{j} \zeta_{j}}
\end{array}\right]=\left[\begin{array}{cccc}
0 & 0 & \cdots & 0 \\
1 & 0 & \cdots & 0 \\
\vdots & \vdots & \cdots & \vdots \\
0 & 0 & \cdots & 0 \\
0 & 0 & \cdots & 1
\end{array}\right]\left[\begin{array}{c}
z_{k}^{\zeta_{j}, 1} \\
z_{k}^{\zeta_{j}, 2} \\
\vdots \\
z_{k}^{\zeta_{j} \zeta_{j}}
\end{array}\right],
$$

or, equivalently,

$$
\begin{aligned}
& \nabla_{0}^{n} z_{k}^{\zeta_{j}, 1}=0, \\
& \nabla_{0}^{n} z_{k}^{\zeta_{j}, 2}=z_{k}^{\zeta_{j}, 1}, \\
& \vdots \\
& \nabla_{0}^{n} z_{k}^{\zeta_{j}, \zeta_{j}-1}=z_{k}^{\zeta_{j} \zeta_{j}-2}, \\
& \nabla_{0}^{n} z_{k}^{\zeta_{j} \zeta_{j}}=z_{k}^{\zeta_{j}, \zeta_{j}-1}, \\
& 0=z_{k}^{\zeta_{j}, \zeta_{j}} .
\end{aligned}
$$


We have a system of $\zeta_{j}+1$ difference equations and $\zeta_{j}$ unknowns. Starting from the last equation, we get the solutions

$$
\begin{aligned}
& z_{k}^{\zeta_{j}, \zeta_{j}}=0, \\
& z_{k}^{\zeta_{j}, \zeta_{j}-1}=0, \\
& z_{k}^{\zeta_{j} \zeta_{j}-2}=0, \\
& \vdots \\
& z_{k}^{\zeta_{j}, 1}=0,
\end{aligned}
$$

which means that the solution of the system (32) is unique and is the zero solution. The proof is completed.

Proposition 3.3 The subsystem (33) has an infinite number of solutions that can be taken arbitrarily

$$
Z_{k}^{g}=C_{k, 2}
$$

Proof It is easy to observe that the subsystem

$$
0_{h, g} \cdot \nabla_{0}^{n} Z_{k+1}^{g}=0_{h, g} \cdot Z_{k}^{g}
$$

does not provide any non-zero equations. Hence all its solutions can be taken arbitrarily. The proof is completed.

We can now state the following theorem.

Theorem 3.1 Consider the system (1) with a singular pencil and known boundary conditions of type (2). Then the boundary value problem (1)-(2) is consistent if and only if:

1.

$$
\left\|J_{p}\right\|<1
$$

2. the column minimal indices are zero, i.e.,

$$
\operatorname{dim} \mathcal{N}_{r}(s F-G)=0
$$

3.

$$
\left[\begin{array}{l}
B_{1} \\
B_{2}
\end{array}\right] \in \operatorname{colspan}\left[\begin{array}{c}
A_{1} Q_{p} \\
A_{2} Q_{p}(N+1)^{\frac{\overline{n-1}}{1}} F_{n, n}\left(J_{p}(N+n)^{\bar{n}}\right)\left(I_{p}-J_{p}\right)
\end{array}\right] .
$$

Furthermore, when the boundary value problem (1)-(2) is consistent, it has a unique solution if and only if 
1.

$$
p \leq r_{1}+r_{2}
$$

2.

$$
\operatorname{rank}\left[\begin{array}{c}
A_{1} Q_{p} \\
A_{2} Q_{p}(N+1)^{\overline{n-1}} F_{n, n}\left(I_{p}(N+n)^{\bar{n}}\right)\left(I_{p}-J_{p}\right)
\end{array}\right]=p .
$$

In this case the unique solution is given by the formula

$$
Y_{k}=Q_{p}(k+1)^{\overline{n-1}} F_{n, n}\left(J_{p}(k+n)^{\bar{n}}\right)\left(I_{p}-J_{p}\right) C,
$$

where $C$ is the unique solution of the algebraic system

$$
\left[\begin{array}{c}
A_{1} Q_{p} \\
A_{2} Q_{p}(N+1)^{\overline{n-1}} F_{n, n}\left(J_{p}(N+n)^{\bar{n}}\right)\left(I_{p}-J_{p}\right)
\end{array}\right] C=\left[\begin{array}{l}
B_{1} \\
B_{2}
\end{array}\right] .
$$

In any other case the system has infinite solutions.

Proof First we consider that the system has non-zero column minimal indices and nonzero row minimal indices. By using the transformation (34), the solutions of the subsystems (29), (30), (31), (32) and (33) are given by (10), (12), (36), (40) and (44) respectively. Note that from Proposition 2.1 the solution (10) exists if and only if

$$
\left\|J_{p}\right\|<1
$$

Furthermore, if

$$
Z_{k}=\left[\begin{array}{c}
Z_{k}^{p} \\
Z_{k}^{q} \\
Z_{k}^{\epsilon} \\
Z_{k}^{\zeta} \\
Z_{k}^{g}
\end{array}\right]=\left[\begin{array}{c}
(k+1)^{\overline{n-1}} F_{n, n}\left(J_{p}(k+n)^{\bar{n}}\right)\left(I_{p}-J_{p}\right) Z_{0}^{p} \\
0_{q, 1} \\
C_{k, 1} \\
0_{t-h, 1} \\
C_{k, 2}
\end{array}\right]
$$

Since $Z_{0}^{p}$ is unknown, it can be replaced with the unknown vector $C$. Then

$$
Y_{k}=Q Z_{k}=\left[\begin{array}{lllll}
Q_{p} & Q_{q} & Q_{\epsilon} & Q_{\zeta} & Q_{g}
\end{array}\right]\left[\begin{array}{c}
(k+1)^{\overline{n-1}} F_{n, n}\left(J_{p}(k+n)^{\bar{n}}\right)\left(I_{p}-J_{p}\right) C \\
0_{q, 1} \\
C_{k, 1} \\
0_{t-h, 1} \\
C_{k, 2}
\end{array}\right]
$$

or, equivalently,

$$
Y_{k}=Q_{p}(k+1)^{\overline{n-1}} F_{n, n}\left(J_{p}(k+n)^{\bar{n}}\right)\left(I_{p}-J_{p}\right) C+Q_{\epsilon} C_{k, 1}+Q_{g} C_{k, 2} .
$$


Since $C_{k, 1}$ and $C_{k, 2}$ can be taken arbitrarily, it is clear that the general singular discrete time system for every suitable defined boundary condition has an infinite number of solutions. It is clear that the existence of the column minimal indices is the reason that the systems (31) and consequently (33) exist. These systems as shown in Propositions 3.1 and 3.3 have always infinite solutions. Thus a necessary condition for the system to have a unique solution is not to have any column minimal indices which are equal to

$$
\operatorname{dim} \mathcal{N}_{r}(s F-G)=0 .
$$

In this case the Kronecker canonical form of the pencil $s F-G$ has the following form:

$$
s F_{K}-G_{K}:=s I_{p}-J_{p} \oplus s H_{q}-I_{q} \oplus s F_{\zeta}-G_{\zeta} .
$$

Then the system (1) is divided into three subsystems (29), (30), (32) with solutions (10), (12), (40) respectively. Thus

$$
Y_{k}=Q Z_{k}=\left[\begin{array}{lll}
Q_{p} & Q_{q} & Q_{\zeta}
\end{array}\right]\left[\begin{array}{c}
(k+1)^{\overline{n-1}} F_{n, n}\left(J_{p}(k+n)^{\bar{n}}\right)\left(I_{p}-J_{p}\right) C \\
0_{q, 1} \\
0_{t-h, 1}
\end{array}\right]
$$

or, equivalently,

$$
Y_{k}=Q_{p}(k+1)^{\overline{n-1}} F_{n, n}\left(J_{p}(k+n)^{\bar{n}}\right)\left(I_{p}-J_{p}\right) C .
$$

The solution exists if and only if

$$
A_{1} Y_{0}=B_{1}, \quad A_{2} Y_{N}=B_{2}
$$

or, equivalently,

$$
A_{1} Q_{p} C=B_{1}, \quad A_{2} Q_{p}(N+1)^{\overline{n-1}} F_{n, n}\left(J_{p}(N+n)^{\bar{n}}\right)\left(I_{p}-J_{p}\right) C=B_{2},
$$

or, equivalently,

$$
\left[\begin{array}{c}
A_{1} Q_{p} \\
A_{2} Q_{p}(N+1)^{\frac{\overline{n-1}}{F_{n, n}}}\left(J_{p}(N+n)^{\bar{n}}\right)\left(I_{p}-J_{p}\right)
\end{array}\right] C=\left[\begin{array}{l}
B_{1} \\
B_{2}
\end{array}\right] .
$$

For the above algebraic system, there exists at least one solution if and only if

$$
\left[\begin{array}{l}
B_{1} \\
B_{2}
\end{array}\right] \in \text { colspan }\left[\begin{array}{c}
A_{1} Q_{p} \\
A_{2} Q_{p}(N+1)^{\overline{n-1}} F_{n, n}\left(J_{p}(N+n)^{\bar{n}}\right)\left(I_{p}-J_{p}\right)
\end{array}\right] .
$$

The algebraic system (50) contains $r_{1}+r_{2}$ equations and $p$ unknowns. Hence the solution is unique if and only if

$$
p \leq r_{1}+r_{2}
$$


and

$$
\operatorname{rank}\left[\begin{array}{c}
A_{1} Q_{p} \\
A_{2} Q_{p}(N+1)^{\overline{n-1}} F_{n, n}\left(J_{p}(N+n)^{\bar{n}}\right)\left(I_{p}-J_{p}\right)
\end{array}\right]=p,
$$

where $C$ is then the unique solution of (50). The uniqueness of $C$ can be proved as follows. If we assume that the algebraic system has two solutions $C_{1}$ and $C_{2}$, then

$$
\left[\begin{array}{c}
A_{1} Q_{p} \\
A_{2} Q_{p}(N+1)^{\overline{n-1}} F_{n, n}\left(J_{p}(N+n)^{\bar{n}}\right)\left(I_{p}-J_{p}\right)
\end{array}\right] C_{1}=\left[\begin{array}{l}
B_{1} \\
B_{2}
\end{array}\right]
$$

and

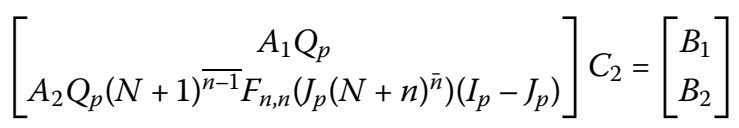

or, equivalently,

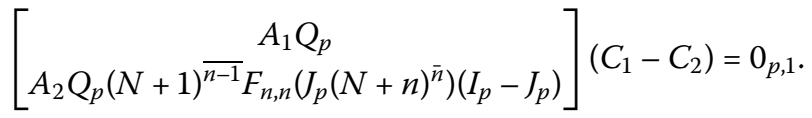

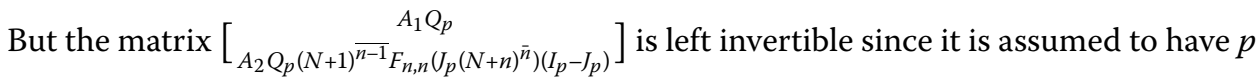
linear independent columns and $r_{1}+r_{2} \geq p$ and hence

$$
C_{1}=C_{2} .
$$

The unique solution is then given from (49). The proof is completed.

\section{Numerical examples}

\section{Example 1}

Assume the system (1) for $k=0,1,2,3$ and $n=\frac{3}{2}$. Let

$$
F=\left[\begin{array}{ccccc}
0 & 1 & 0 & 0 & 0 \\
0 & 0 & 0 & 0 & -1 \\
1 & -1 & 0 & 1 & -1 \\
1 & -1 & 0 & 2 & -1 \\
0 & 0 & 0 & 0 & 0
\end{array}\right]
$$

and

$$
G=\left[\begin{array}{ccccc}
0 & 0 & 1 & -1 & 1 \\
0 & 0 & 0 & 0 & -\frac{1}{4} \\
\frac{1}{2} & -\frac{1}{2} & 0 & -\frac{1}{2} & \frac{1}{2} \\
\frac{1}{2} & -\frac{1}{2} & 1 & -\frac{1}{2} & \frac{3}{4} \\
0 & 0 & 0 & 1 & -1
\end{array}\right] .
$$


Then $\operatorname{det}(s F-G)=\left(s-\frac{1}{2}\right) s\left(s-\frac{1}{4}\right)$ and the pencil is regular. We assume the boundary conditions (2) with

$$
\begin{aligned}
A_{1} & =\left[\begin{array}{lllll}
1 & 0 & 0 & 0 & 0 \\
0 & 1 & 0 & 0 & 0 \\
0 & 0 & 1 & 0 & 0 \\
0 & 0 & 0 & 0 & 1
\end{array}\right], \\
A_{2} & =\left[\begin{array}{lllll}
1 & 0 & 0 & 0 & 1
\end{array}\right], \\
B_{1} & =\left[\begin{array}{c}
0 \\
0 \\
36 \\
36
\end{array}\right]
\end{aligned}
$$

and

$$
B_{2}=24 .
$$

The three finite eigenvalues $(p=3)$ of the pencil are $\frac{1}{2}, 0, \frac{1}{4}$, and the Jordan matrix $J_{p}$ has the form

$$
J_{p}=\left[\begin{array}{ccc}
\frac{1}{2} & 0 & 0 \\
0 & 0 & 0 \\
0 & 0 & \frac{1}{4}
\end{array}\right] .
$$

It is easy to observe that

$$
\left\|J_{p}\right\|<1
$$

By calculating the eigenvectors of the finite eigenvalues, we get the matrix $Q_{p}$

$$
Q_{p}=\left[\begin{array}{lll}
1 & 1 & 0 \\
0 & 1 & 0 \\
0 & 0 & 0 \\
0 & 0 & 1 \\
0 & 0 & 1
\end{array}\right]
$$

Moreover,

$$
A_{1} Q_{p}=\left[\begin{array}{lll}
1 & 1 & 0 \\
0 & 1 & 0 \\
0 & 0 & 1 \\
0 & 0 & 1
\end{array}\right] .
$$

From (9) we get the Mittag-Leffler function

$$
F_{\frac{3}{2}, \frac{3}{2}}\left(J_{p}\left(3+\frac{3}{2}\right)^{\frac{\overline{3}}{2}}\right)=\sum_{i=0}^{\infty} J_{p}^{i} \frac{\left(3+\frac{3}{2}\right)^{\overline{i \frac{3}{2}}}}{\Gamma\left((i+1) \frac{3}{2}\right)}
$$


or, equivalently,

$$
F_{\frac{3}{2}, \frac{3}{2}}\left(J_{p}\left(3+\frac{3}{2}\right)^{\frac{\overline{3}}{2}}\right)=\sum_{i=0}^{\infty} J_{p}^{i} \frac{\Gamma\left(3+\frac{3}{2}(i+1)\right)}{\Gamma\left(3+\frac{3}{2}\right) \Gamma\left((i+1) \frac{3}{2}\right)},
$$

or, equivalently,

$$
F_{\frac{3}{2}, \frac{3}{2}}\left(J_{p}\left(3+\frac{3}{2}\right)^{\frac{\overline{3}}{2}}\right)=\sum_{i=0}^{\infty} J_{p}^{i} \frac{\left(2+\frac{3}{2}(i+1)\right)\left(1+\frac{3}{2}(i+1)\right)\left(\frac{3}{2}(i+1)\right) \Gamma\left(\frac{3}{2}(i+1)\right)}{\Gamma\left(3+\frac{3}{2}\right) \Gamma\left((i+1) \frac{3}{2}\right)},
$$

or, equivalently,

$$
F_{\frac{3}{2}, \frac{3}{2}}\left(J_{p}\left(3+\frac{3}{2}\right)^{\frac{3}{2}}\right)=\frac{1}{\Gamma\left(3+\frac{3}{2}\right)} \sum_{i=0}^{\infty} J_{p}^{i}\left(2+\frac{3}{2}(i+1)\right)\left(1+\frac{3}{2}(i+1)\right)\left(\frac{3}{2}(i+1)\right)
$$

and since $\left\|J_{p}\right\|<1$, by using the sum $\sum_{i=0}^{\infty} x=(1-x)^{-1}$ for $\|x\|<1$, we calculate the sum of the matrix power series $\sum_{i=0}^{\infty} J_{p}^{i}\left(2+\frac{3}{2}(i+1)\right)\left(1+\frac{3}{2}(i+1)\right)\left(\frac{3}{2}(i+1)\right)$, and we get

$$
F_{\frac{3}{2}, \frac{3}{2}}\left(J_{p}\left(3+\frac{3}{2}\right)^{\frac{\overline{3}}{2}}\right)=\frac{1}{\Gamma\left(3+\frac{3}{2}\right)}\left(-\frac{1}{9} J_{p}^{2}+\frac{20}{9} J_{p}+\frac{35}{9} I_{p}\right)\left(I_{p}-J_{p}\right)^{-4} .
$$

And since

$$
A_{2} Q_{p}=\left[\begin{array}{lll}
1 & 1 & 1
\end{array}\right]
$$

by using the above expression

$$
A_{2} Q_{p}(4)^{\frac{1}{2}} F_{\frac{3}{2}, \frac{3}{2}}\left(J_{p}\left(3+\frac{3}{2}\right)^{\frac{\overline{3}}{2}}\right)\left(I_{p}-J_{p}\right)=\frac{1}{36}\left[\begin{array}{lll}
1 & 1 & 1
\end{array}\right]\left[\begin{array}{ccc}
358 & 0 & 0 \\
0 & 35 & 0 \\
0 & 0 & 24
\end{array}\right]
$$

or, equivalently,

$$
A_{2} Q_{p}(4)^{\frac{1}{2}} F_{\frac{3}{2}, \frac{3}{2}}\left(J_{p}\left(3+\frac{3}{2}\right)^{\frac{\overline{3}}{2}}\right)\left(I_{p}-J_{p}\right)=\frac{1}{36}\left[\begin{array}{lll}
358 & 35 & 24
\end{array}\right],
$$

it is easy to observe that the conditions (13), (14) and (15) are satisfied. Thus from Theorem 2.1 the unique solution of the boundary value problem (1)-(2) is given by

$$
Y_{k}=Q_{p}(k+1)^{\frac{3}{2}-1} F_{\frac{3}{2}, \frac{3}{2}}\left(J_{p}\left(k+\frac{3}{2}\right)^{\frac{\overline{3}}{2}}\right)\left(I_{p}-J_{p}\right) C
$$

or, equivalently, by

$$
Y_{k}=\frac{1}{\Gamma(k+1)} \sum_{i=0}^{\infty} \frac{\Gamma\left(k+\frac{3}{2}(i+1)\right)}{\Gamma\left(\frac{3}{2}(i+1)\right)}\left[\begin{array}{ccc}
\frac{1}{2^{i+1}} & 0 & 0 \\
0 & 0 & 0 \\
0 & 0 & 0 \\
0 & 0 & \frac{3}{4^{i+1}} \\
0 & 0 & \frac{3}{4^{i+1}}
\end{array}\right] C,
$$


where $C$ is the unique solution of the algebraic system

$$
\left[\begin{array}{c}
A_{1} Q_{p} \\
A_{2} Q_{p}(4)^{\frac{1}{2}} F_{\frac{3}{2}, \frac{3}{2}}\left(J_{p}\left(3+\frac{3}{2}\right)^{\frac{3}{2}}\right)\left(I_{p}-J_{p}\right)
\end{array}\right] C=\left[\begin{array}{l}
B_{1} \\
B_{2}
\end{array}\right]
$$

or, equivalently,

$$
C=\left[\begin{array}{c}
0 \\
0 \\
36
\end{array}\right]
$$

and thus the unique solution of the boundary value problem is

$$
Y_{k}=\frac{27}{\Gamma(k+1)} \sum_{i=0}^{\infty} \frac{\Gamma\left(k+\frac{3}{2}(i+1)\right)}{\Gamma\left(\frac{3}{2}(i+1)\right)}\left[\begin{array}{c}
0 \\
0 \\
0 \\
\frac{1}{4^{i}} \\
\frac{1}{4^{i}}
\end{array}\right] .
$$

\section{Example 2}

We assume the system (1) as in Example 1 but with different boundary conditions. Let

$$
\begin{aligned}
A_{1} & =\left[\begin{array}{lllll}
1 & 0 & 0 & 0 & 0 \\
0 & 1 & 0 & 0 & 0 \\
0 & 0 & 1 & 0 & 0 \\
0 & 0 & 0 & 0 & 1
\end{array}\right], \\
A_{2} & =\left[\begin{array}{lllll}
1 & 0 & 0 & 0 & 1
\end{array}\right], \\
B_{1} & =\left[\begin{array}{c}
0 \\
0 \\
0 \\
36
\end{array}\right]
\end{aligned}
$$

and

$$
B_{2}=24 .
$$

It is easy to observe that

$$
\left[\begin{array}{l}
B_{1} \\
B_{2}
\end{array}\right] \notin \operatorname{colspan}\left[\begin{array}{c}
A_{1} Q_{p} \\
A_{2} Q_{p}(4)^{\frac{\overline{1}}{2}} F_{\frac{3}{2}, \frac{3}{2}}\left(J_{p}\left(3+\frac{3}{2}\right)^{\frac{\overline{3}}{2}}\right)\left(I_{p}-J_{p}\right)
\end{array}\right]
$$

since

$$
\left[\begin{array}{c}
0 \\
0 \\
0 \\
36 \\
24
\end{array}\right] \notin \text { colspan }\left[\begin{array}{ccc}
36 & 36 & 0 \\
0 & 36 & 0 \\
0 & 0 & 36 \\
0 & 0 & 36 \\
358 & 35 & 24
\end{array}\right]
$$


and thus from Theorem 2.1, and since (13) does not hold, the boundary value problem is not consistent.

\section{Example 3}

Consider the system (1) and let

$$
F=\left[\begin{array}{lllll}
1 & 1 & 1 & 1 & 1 \\
0 & 1 & 1 & 0 & 1 \\
1 & 1 & 1 & 1 & 1 \\
0 & 1 & 1 & 0 & 1 \\
1 & 0 & 1 & 0 & 0 \\
0 & 0 & 1 & 1 & 1
\end{array}\right], \quad G=\left[\begin{array}{lllll}
1 & 2 & 2 & 1 & 2 \\
0 & 2 & 2 & 0 & 2 \\
1 & 2 & 2 & 2 & 3 \\
0 & 2 & 3 & 1 & 3 \\
0 & 0 & 0 & 0 & 0 \\
1 & 0 & 1 & 0 & 0
\end{array}\right]
$$

Since the matrices $F, G$ are non-square, the matrix pencil $s F-G$ is singular and has invariants such as the finite elementary divisors $s-2, s-1$, an infinite elementary divisor of degree 1 and the row minimal indices $\zeta_{1}=0, \zeta_{2}=1$. Since the Jordan matrix has the form

$$
J_{p}=\left[\begin{array}{ll}
2 & 0 \\
0 & 1
\end{array}\right]
$$

with

$$
\left\|J_{p}\right\|>1
$$

for every induced matrix norm, from Theorem 3.1 the boundary value problem (1)-(2) is non-consistent.

\section{Example 4}

Consider the system (1) for $k=0,1,2,3$ and $n=\frac{3}{2}$. Let

$$
F=\left[\begin{array}{ll}
1 & 0 \\
0 & 1 \\
0 & 0
\end{array}\right]
$$

and

$$
G=\left[\begin{array}{cc}
\frac{3}{4} & 0 \\
0 & 0 \\
0 & 1
\end{array}\right]
$$

Since the matrices $F, G$ are non-square, the matrix pencil $s F-G$ is singular and has invariants such as a finite elementary divisor $s-\frac{3}{4}$ and the row minimal indices $\zeta_{1}=0, \zeta_{2}=1$. We assume the boundary conditions (2) with

$$
A_{1}=\left[\begin{array}{ll}
1 & 1
\end{array}\right], \quad A_{2}=\left[\begin{array}{ll}
0 & 1 \\
0 & 2
\end{array}\right], \quad B_{1}=2
$$


and

$$
B_{2}=\left[\begin{array}{l}
0 \\
0
\end{array}\right]
$$

The Jordan matrix is $J_{p}=\frac{3}{4}$ with $\left\|J_{p}\right\|<1$ for every induced matrix norm. By calculating the matrix $Q_{p}$, we get

$$
Q_{p}=\left[\begin{array}{l}
1 \\
0
\end{array}\right]
$$

Moreover,

$$
A_{1} Q_{p}=1,
$$

and since

$$
A_{2} Q_{p}=\left[\begin{array}{l}
0 \\
0
\end{array}\right] \text {, }
$$

we get

$$
A_{2} Q_{p}(4)^{\frac{\overline{1}}{2}} F_{\frac{3}{2}, \frac{3}{2}}\left(J_{p}\left(3+\frac{3}{2}\right)^{\frac{\overline{3}}{2}}\right)\left(I_{p}-J_{p}\right)=\left[\begin{array}{l}
0 \\
0
\end{array}\right] .
$$

By using (59), (60), it is easy to observe that the conditions (45), (46), (47) and (48) are satisfied and thus from Theorem 3.1 the unique solution of the boundary value problem (1)-(2) is given by

$$
Y_{k}=Q_{p}(k+1)^{\frac{\overline{1}}{2}} F_{\frac{3}{2}, \frac{3}{2}}\left(J_{p}\left(k+\frac{3}{2}\right)^{\frac{3}{2}}\right)\left(I_{p}-J_{p}\right) C
$$

or, equivalently, by

$$
Y_{k}=\frac{1}{\Gamma(k+1)} \sum_{i=0}^{\infty} \frac{\Gamma\left(k+\frac{3}{2}(i+1)\right)}{\Gamma\left(\frac{3}{2}(i+1)\right)}\left[\begin{array}{c}
3^{i} \\
4^{i+1} \\
0
\end{array}\right] C,
$$

where $C$ is the unique solution of the algebraic system

$$
\left[\begin{array}{c}
A_{1} Q_{p} \\
A_{2} Q_{p}(4)^{\frac{1}{2}} F_{\frac{3}{2}, \frac{3}{2}}\left(J_{p}\left(3+\frac{3}{2}\right)^{\frac{\overline{3}}{2}}\right)\left(I_{p}-J_{p}\right)
\end{array}\right] C=\left[\begin{array}{l}
B_{1} \\
B_{2}
\end{array}\right]
$$

or, equivalently,

$$
C=2 \text {, }
$$


and thus the unique solution of the boundary value problem is

$$
Y_{k}=\frac{2}{\Gamma(k+1)} \sum_{i=0}^{\infty} \frac{\Gamma\left(k+\frac{3}{2}(i+1)\right)}{\Gamma\left(\frac{3}{2}(i+1)\right)}\left[\begin{array}{c}
\frac{3^{i}}{4^{i+1}} \\
0
\end{array}\right] \text {. }
$$

\section{Conclusions}

In this article, we study the boundary value problem of a class of a singular system of fractional nabla difference equations whose coefficients are constant matrices. By taking into consideration the cases that the matrices are square with the leading coefficient singular, square with an identically zero matrix pencil and non-square, we study the conditions under which the boundary value problem has unique, infinite and no solutions. Furthermore, we provide a formula for the case of the unique solution. As a further extension of this article, one can study the stability, the behavior under perturbation and possible applications in economics and engineering of singular matrix difference/differential equations of fractional order. For all this, there is already some research in progress.

\section{Competing interests}

The authors declare that they have no competing interests.

\section{Authors' contributions}

IKD wrote the first draft of the manuscript and DB correct it and prepared the final version of it. All authors read and approved the final manuscript.

\section{Author details \\ 'School of Mathematics and Maxwell Institute, The University of Edinburgh, Mayfield Road, Edinburgh, EH9 3JZ, United Kingdom. ${ }^{2}$ Department of Mathematics and Computer Sciences, Cankaya University, Ankara, Turkey. ${ }^{3}$ Institute of Space Sciences, Magurele, Bucharest, Romania. ${ }^{4}$ Department of Chemical and Materials Engineering, Faculty of Engineering, King Abdulaziz University, Jeddah, Saudi Arabia.}

\section{Acknowledgements}

We would like to express our sincere gratitude to Professor Gl Kalogeropoulos for his helpful and fruitful discussions that clearly improved this article. Moreover, we are very grateful to the anonymous referees for their valuable suggestions that improved the article.

\section{Received: 20 February 2013 Accepted: 18 May 2013 Published: 19 June 2013}

\section{References}

1. Baleanu, D, Diethelm, K, Scalas, E: Fractional Calculus: Models and Numerical Methods. World Scientific, Singapore (2012)

2. Glockle, WG, Nonnenmacher, TF: A fractional calculus approach to self-similar protein dynamics. Biophys. J. 68(1), 46-53 (1995)

3. Hilfe, R (ed.): Applications of Fractional Calculus in Physics. World Scientific, River Edge (2000)

4. Kaczorek, T: Selected Problems of Fractional Systems Theory, vol. 411. Springer, Berlin (2011)

5. Malinowska, AB, Torres, DFM: Introduction to the Fractional Calculus of Variations. Imperial College Press, London (2012). ISBN:978-1-84816-966-1/hbk

6. Metzler, R, Schick, W, Kilian, HG, Nonnenmacher, TF: Relaxation in filled polymers: a fractional calculus approach J. Chem. Phys. 103(16), 7180-7186 (1995)

7. Podlubny, I: Fractional Differential Equations. Mathematics in Science and Engineering. Academic Press, San Diego (1999)

8. Klamka, J: Controllability and minimum energy control problem of fractional discrete-time systems. In: New Trends in Nanotechnology and Fractional Calculus, pp. 503-509. Springer, New York (2010)

9. Agarwal, RP, Benchohra, M, Hamani, S: A survey on existence results for boundary value problems of nonlinear fractional differential equations and inclusions. Acta Appl. Math. 109(3), 973-1033 (2010)

10. Ahrendt, K, Castle, L, Holm, M, Yochman, K: Laplace transforms for the nabla-difference operator and a fractional variation of parameters formula. Commun. Appl. Anal. (2011)

11. Atici, FM, Eloe, PW: Linear systems of fractional nabla difference equations. Rocky Mt. J. Math. 41(2), $353-370$ (2011)

12. Atici, FM, Eloe, PW: Initial value problems in discrete fractional calculus. Proc. Am. Math. Soc. 137(3), 981-989 (2009)

13. Baleanu, D, Mustafa, OG, Agarwal, RP: Asymptotically linear solutions for some linear fractional differential equations. Abstr. Appl. Anal. 2010, Article ID 865139 (2010)

14. Baleanu, D, Mustafa, OG: On the global existence of solutions to a class of fractional differential equations. Comput. Math. Appl. 59(5), 1835-1841 (2010)

15. Baleanu, D, Babakhani, A: Employing of some basic theory for class of fractional differential equations. Adv. Differ. Equ. 2011, Article ID 296353 (2011) 
16. Bastos, NRO, Ferreira, RAC, Torres, DFM: Necessary optimality conditions for fractional difference problems of the calculus of variations. Discrete Contin. Dyn. Syst. 29(2), 417-437 (2011)

17. Bastos, NRO, Ferreira, RAC, Torres, DFM: Discrete-time fractional variational problems. Signal Process. 91 (3), 513-524 (2011)

18. Bastos, NRO, Mozyrska, D, Torres, DFM: Fractional derivatives and integrals on time scales via the inverse generalized Laplace transform. Int. J. Math. Comput. 11, 1-9 (2011)

19. Debbouche, A: Fractional nonlocal impulsive quasilinear multi-delay integro-differential systems. Adv. Differ. Equ. 2011, 5 (2011)

20. Debbouche, A, Baleanu, D: Controllability of fractional evolution nonlocal impulsive quasilinear delay integro-differential systems. Comput. Math. Appl. 62(3), 1442-1450 (2011)

21. Debbouche, A, Baleanu, D, Agarwal, RP: Nonlocal nonlinear integrodifferential equations of fractional orders. Adv. Differ. Equ. 2012, 78 (2012)

22. Ferreira, RAC, Torres, DFM: Fractional $h$-difference equations arising from the calculus of variations. Appl. Anal. Discrete Math. 5(1), 110-121 (2011)

23. Hein, J, McCarthy, Z, Gaswick, N, McKain, B, Speer, K: Laplace transforms for the nabla-difference operator. Panam. Math. J. 21(3), 79-97 (2011)

24. Kaczorek, T: Positive stable realizations of fractional continuous-time linear systems. Int. J. Appl. Math. Comput. Sci. 21(4), 697-702 (2011)

25. Kaczorek, T: Application of the Drazin inverse to the analysis of descriptor fractional discrete-time linear systems with regular pencils. Int. J. Appl. Math. Comput. Sci. 23(1), 29-33 (2013)

26. Klamka, J: Controllability of dynamical systems. Mat. Stosow. 50(9), 57-75 (2008)

27. Klamka, J, Wyrwał, J: Controllability of second-order infinite-dimensional systems. Syst. Control Lett. 57(5), 386-391 (2008)

28. Dai, L: Singular Control Systems. Lecture Notes in Control and Information Sciences (1988) (edited by M Thoma and A Wyner)

29. Dassios, IK: On non homogeneous linear generalized linear discrete time systems. Circuits Syst. Signal Process. 31(5), 1699-1712 (2012)

30. Dassios, IK, Kalogeropoulos, G: On a non-homogeneous singular linear discrete time system with a singular matrix pencil. Circuits Syst. Signal Process. (2013). doi:10.1007/s00034-012-9541-8

31. Dassios, I: On solutions and algebraic duality of generalized linear discrete time systems. Discrete Math. Appl. 22(5-6), 665-682 (2012)

32. Dassios, I: On stability and state feedback stabilization of singular linear matrix difference equations. Adv. Differ. Equ. 2012, 75 (2012)

33. Dassios, I: On robust stability of autonomous singular linear matrix difference equations. Appl. Math. Comput. 218(12), 6912-6920 (2012)

34. Dassios, I: On a boundary value problem of a class of generalized linear discrete time systems. Adv. Differ. Equ. 2011, $51(2011)$

35. Grispos, E, Giotopoulos, S, Kalogeropoulos, G: On generalised linear discrete-time regular delay systems. J. Inst. Math. Comput. Sci., Math. Ser. 13(2), 179-187 (2000)

36. Grispos, E, Kalogeropoulos, G, Mitrouli, M: On generalised linear discrete-time singular delay systems. In: Lipitakis, EA (ed.) Proceedings of the 5th Hellenic-European Conference on Computer Mathematics and Its Applications (HERCMA 2001), Athens, Greece, September 20-22 2001, 2 volumes, pp. 484-486. LEA, Athens (2002)

37. Grispos, E, Kalogeropoulos, G, Stratis, I: On generalised linear discrete-time singular delay systems. J. Math. Anal. Appl. 245(2), 430-446 (2000)

38. Grispos, E: Singular generalised autonomous linear differential systems. Bull. Greek Math. Soc. 34, 25-43 (1992)

39. Kalogeropoulos, GI: Matrix pencils and linear systems. PhD thesis, City University, London (1985)

40. Kalogeropoulos, G, Stratis, IG: On generalized linear regular delay systems. J. Math. Anal. Appl. 237(2), 505-514 (1999)

41. Karcanias, N, Kalogeropoulos, G: Geometric theory and feedback invariants of generalized linear systems: a matrix pencil approach. Circuits Syst. Signal Process. 8(3), 375-397 (1989)

42. Rugh, WJ: Linear System Theory. Prentice Hall International, London (1996)

43. Sandefur, JT: Discrete Dynamical Systems. Academic Press, San Diego (1990)

44. Gantmacher, RF: The Theory of Matrices. Vols. I, II. Chelsea, New York (1959)

45. Mitrouli, M, Kalogeropoulos, G: A compound matrix algorithm for the computation of the Smith form of a polynomial matrix. Numer. Algorithms 7(2-4), 145-159 (1994)

46. Nagai, A: Discrete Mittag-Leffler function and its applications. Publ. Res. Inst. Math. Sci. 1302, 1-20 (2003)

doi:10.1186/1687-2770-2013-148

Cite this article as: Dassios and Baleanu: On a singular system of fractional nabla difference equations with boundary conditions. Boundary Value Problems 2013 2013:148. 